\title{
A modeling and performance of the triple field plate HEMT
}

\author{
Kourdi Zakarya $^{1}$, Abdelkhader Hamdoun ${ }^{2}$ \\ ${ }^{1}$ GAS Department, Center satellites developments, Agency Space Algerian, Algeria \\ ${ }^{2}$ Electronic Department, University of Tlemcen, Algeria
}

\begin{tabular}{|c|c|}
\hline Article Info & ABSTRACT \\
\hline Article history: & \multirow{6}{*}{$\begin{array}{l}\text { We present this work by two steps. In the first one, the new structure } \\
\text { proposed of the FP-HEMTs device (Field plate High Electron Mobility } \\
\text { Transistor) with a T-gate on an 4H-SIC substrate to optimize these electrical } \\
\text { performances, multiple field-plates were used with aluminum oxide to split } \\
\text { the single electric field peak into several smaller peaks, and as passivation } \\
\text { works to reduce scaling leakage current. In the next, we include a modeling } \\
\text { of a simulation in the Tcad-Silvaco Software for realizing the study of the } \\
\text { influence of negative voltage applied to gate T-shaped in OFF state time and } \\
\text { high power with ambient temperature, the performance differences between } \\
\text { the 3FP and the SFP devices are discussed in detail. }\end{array}$} \\
\hline Received Jul 25, 2018 & \\
\hline Revised Sept 28, 2018 & \\
\hline Accepted Oct 12, 2018 & \\
\hline Keywords: & \\
\hline $\begin{array}{l}\text { Field plate } \\
\text { HEMT }\end{array}$ & \\
\hline
\end{tabular}

Copyright (C) 2019 Institute of Advanced Engineering and Science.

Tcad-silvaco

All rights reserved.

\section{Corresponding Author:}

Kourdi Zakarya,

GAS Department, Center satellites developments

Agency Space Algerian,

POS 50 ILOT T12 Bir El Djir Oran, Algeria.

Email: zkourdi@hotmail.fr

\section{INTRODUCTION}

Research concerning of a HEMTs device has significant efforts in providing exploring new solution for optimal performance with several domains searches very active. The innovative technology of this device type can also be attributed to the adoption with novel structures and analysis operating principles process [1], such as the dynamically doping/dedoping [2]. The application of the high breakdown field effect [3], [4]. The team has been created different structure for field plates on GaN HEMT slant structures [5], 2nd plate [6], such as Field plates (FPs), including source FPs (SFPs) [7], gate FPs (GFPs)[8], and multi-FPs (MFPs) [9], [10], [11]. Modeling a new structure as possible as of the next generation of device creative structure in the next generation of the material III-N has interest in this research, Simulation Tcad-Silvaco software program and superficial genetic algorithm [12] can help for private analysis a new proposed structure. The accurate results process examination needs many model's physics approach can be delivered by a hydrodynamic or quantum model [13].

The main features of our model as proposed in this work a quantum mobility models for the peculiarities of the III-N material process system [14]. A software simulation used for implemented calibrated setup in a device from a recent generation of a HEMT with adapts physics models. A high precision for all relevant characteristics was achieved.

At the moment, Modern methods used for describing the device characteristics keep abreast of rapid and effective progress in devising solutions and minimizing the margin of uncertainty be limited and reduce the time of finding the optimum data. Among the semi-classical models and Monte Carlo method offer the detailed explanation of the solution but is limited in realistic engineering applications for its computational expenses [15].

All elements intrinsic, extrinsic and parasitic of device (due to connecting wires, metallic contacts, packaging, etc.) are very important index in high frequency, in this case a small signal circuit equivalent can't 
be become comparable with the parasitic parameters. We have calibrated simulation a base of the calculate these values without any discrepancy in neglecting or inaccurately. To accurately solve a problem with parasitic elements, in order to provide a more accurate representation of the parasitic elements, a problem need the emphasis in solve by the rendering device performance has affected by many simultaneous factors [16].

A solution for thus enhancing the device performance and their extreme thinness alleviate shortchannel effects with this technology [17], the fabrication of double gate device which need complicated technology and has been not deserving a complete special attention irrespective has been very expensive [18].

While this paper focused on the analysis results a biased of the quantum models, new Monte Carlo method and genetic algorithm for found optimal circuit equivalent has been verified with study all side effect to provide a correct physical insight into the device properties.

\section{DEVICE AND MODELING}

The device is shown in Figure 1(a). The first contact Schottky used Gold "Au" for a wider head Tshaped gate process electrode. Then, source/drain/field plate electrodes were formed by "Au" (250 nm) are chosen for ohmic contacts, the device design features a heterostructure $\operatorname{In}_{0.18} \mathrm{Al}_{0.82} \mathrm{~N} / \mathrm{GaN}$, where the periphery oxide $\mathrm{Al}_{2} \mathrm{O}_{3}$ of the Gate is a difference with conventional designs [19].

This gives rise to a conduction band shape for the barrier that, for the same sheet carrier concentration based on model Fujitsu [20], the Hall mobility and sheet carrier concentration were $1300 \mathrm{~cm}^{2}$ $\mathrm{V}^{-1} \mathrm{~s}^{-1}$ and $1 \times 10^{13} \mathrm{~cm}^{-2}$. The heterojunction features a sheet charge density of $1.85 \times 10^{13} \mathrm{~cm}^{2}$.

The performance of this device been simulated by Tcad-Silvaco software. We use two steps for simulated this device: The first step focused to create a structure in the framework DevEdit. The second step focuses to analyze this structure in the framework Atlas system.

We see in the Figure 1(b) a cross section of the structure, it's located over the layer of substrate $(4 \mathrm{H}-$ $\mathrm{SiC})$. We present each the layer in the flow: We find that the color gold region corresponds to the electrodes (i.e. the field plate, source, drain and gate), the color brown corresponds to layers of the channel and cap layer, the color red corresponds to layers of a donor and Schottky, the buffer and spacer layers corresponds to green color and finally gray color regions correspond to the substrate.
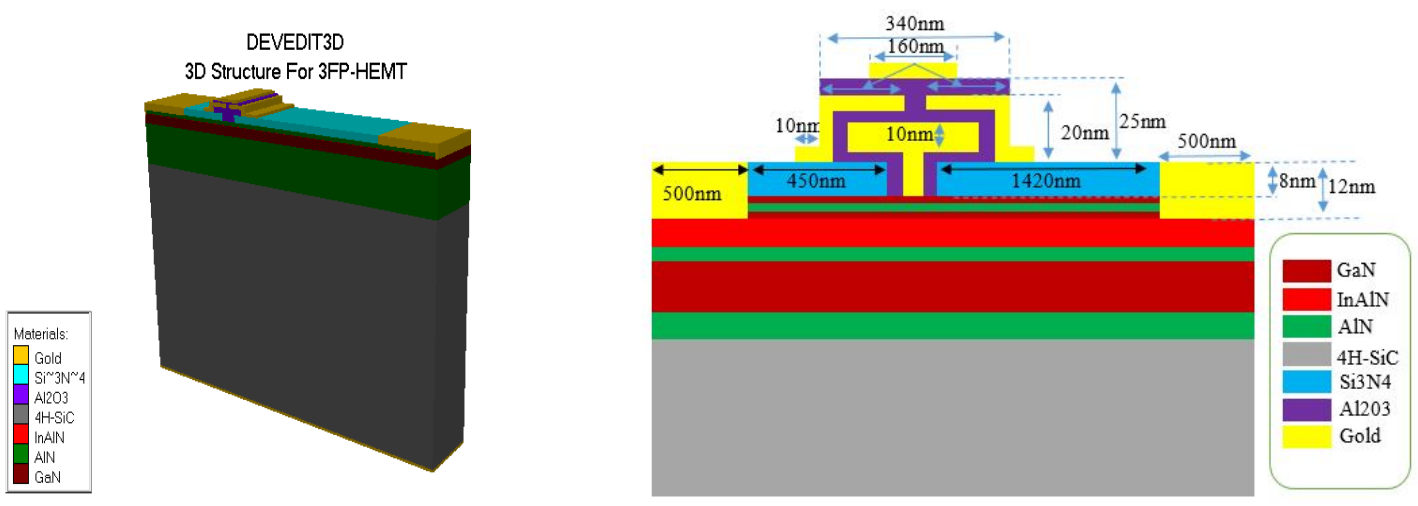

Figure 1. View structure HEMT InAIN/GaN triple field plate in left shown 3D triple FP-HEMT in silvacotcad and in other side of the longitudinal section of the device

While in this paper is focus on illustration design the HEMT structure device proposed for the optimization process using a genetic algorithm to extract the device dimensions, you should know that it is through a well-designed structure, you will:

a. Improve the performance with minimizing adverse effect content.

b. Larger safe operating area.

c. High switching speed.

It is therefore possible to calibrate the HEMT model using simplified device geometry, so care should be taken to find initial values as close as possible to the optimal calibration point. From the score the optimizer generates a new set of model parameters which are then used for the next simulation run [21]. 


\section{RESULTS AND INTERPETATIONS}

The gate length of the device is $\mathrm{L}_{\mathrm{G}}=15 \mathrm{~nm}$ in this work. We simulated the device with SilvacoTcad software. We demonstrated 3D device modeling of an InAlN/GaN HEMT with quantum correction, which was specified with three different statements. AGDEVICE confines the MC electrons to the $4 \mathrm{H}-\mathrm{SiC}$ substrate.

As a result, the "Electron Conc" and "Average Electron Conc" fields in the devices do not show electrons in the oxide even though electron penetration of the InAlN barrier is considered as part of the model. This choice is conventional. The model reasonably predicts the impact of quantum size effects on the electron concentration and the terminal currents [22].

After the definition of the HEMT structure and materials used for model, the initial solution is obtained from the gate voltage set to zero, the structure under zero bias (the initial case reported for supply voltages), and the solutions are obtained from the choices specified in the algorithm, to obtaining a depth characterization from the simulation.

Accurate simulation results can be obtained by solving quantum genetics model physics for simulation the analyze device performance [23], the device temperature is not at all constant, especially at the gate exit in the reality for that the simulation has been begin $300 \mathrm{~K}$ and this value is higher with time, specialized numerical techniques required for that GIGA model should be used to simulate the heat-flow in the device [24], it's included heat generation, heat flow, lattice heating, heat sinks, and effects of local temperature on physical constants with BLAZE models from Tcad-Silvaco software are coupled through self-consistent calculations [25].

\subsection{DC results}

In this device simulation, the electrical transfer and output characteristics are illustrated in Figure 2. We changed the acceleration of the drain voltage between $0 \underline{\mathrm{V}}$ to $3 \underline{\mathrm{V}}$, when the simulation was first conducted to obtain the I-V characteristic in the DC mode to change the state of the gate voltage by 5 different bias values, $\mathrm{V}_{\mathrm{GS}}=1.0 \underline{\mathrm{V}}$ to $-4.0 \underline{\mathrm{V}}$ with a step of $-1.0 \underline{\mathrm{V}}$ for both HEMTs.

The increasingly positive drain voltage led to the electric field across the channel increasing the speed of the electron. The voltage distribution across the channel led to a voltage difference between the gate and the channel along it, with the transistor demonstrating a variable resistance behavior controlled by the gate voltage and the note is with FP that region is large. This indicates excellent gate control of the 2DEG channel [26], and the maximum drain current available reached $2056 \underline{\mathrm{mA} / \mathrm{mm}}$ in the first and $2450 \underline{\mathrm{mA} / \mathrm{mm}}$ in the other, when $\mathrm{V}_{\mathrm{GS}}$ was biased at $0.0 \underline{\mathrm{V}} \& \mathrm{~V}_{\mathrm{DS}}=3.0 \underline{\mathrm{V}}$.

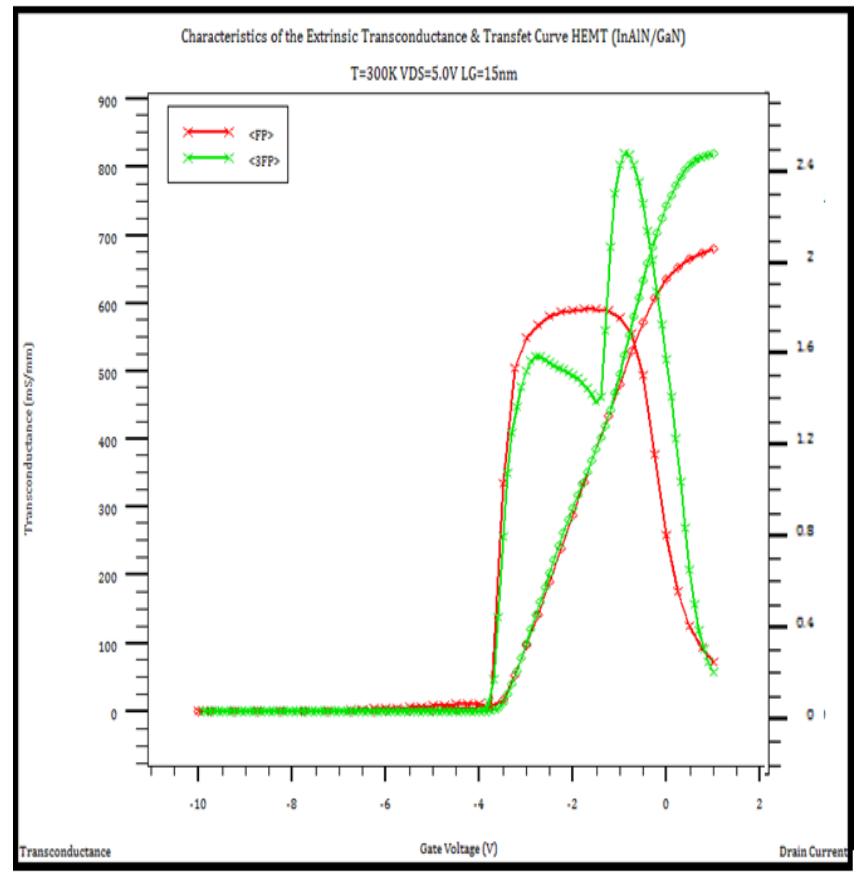

Int J Pow Elec \& Dri Syst, Vol. 10, No. 1, March 2019: 398 - 405 


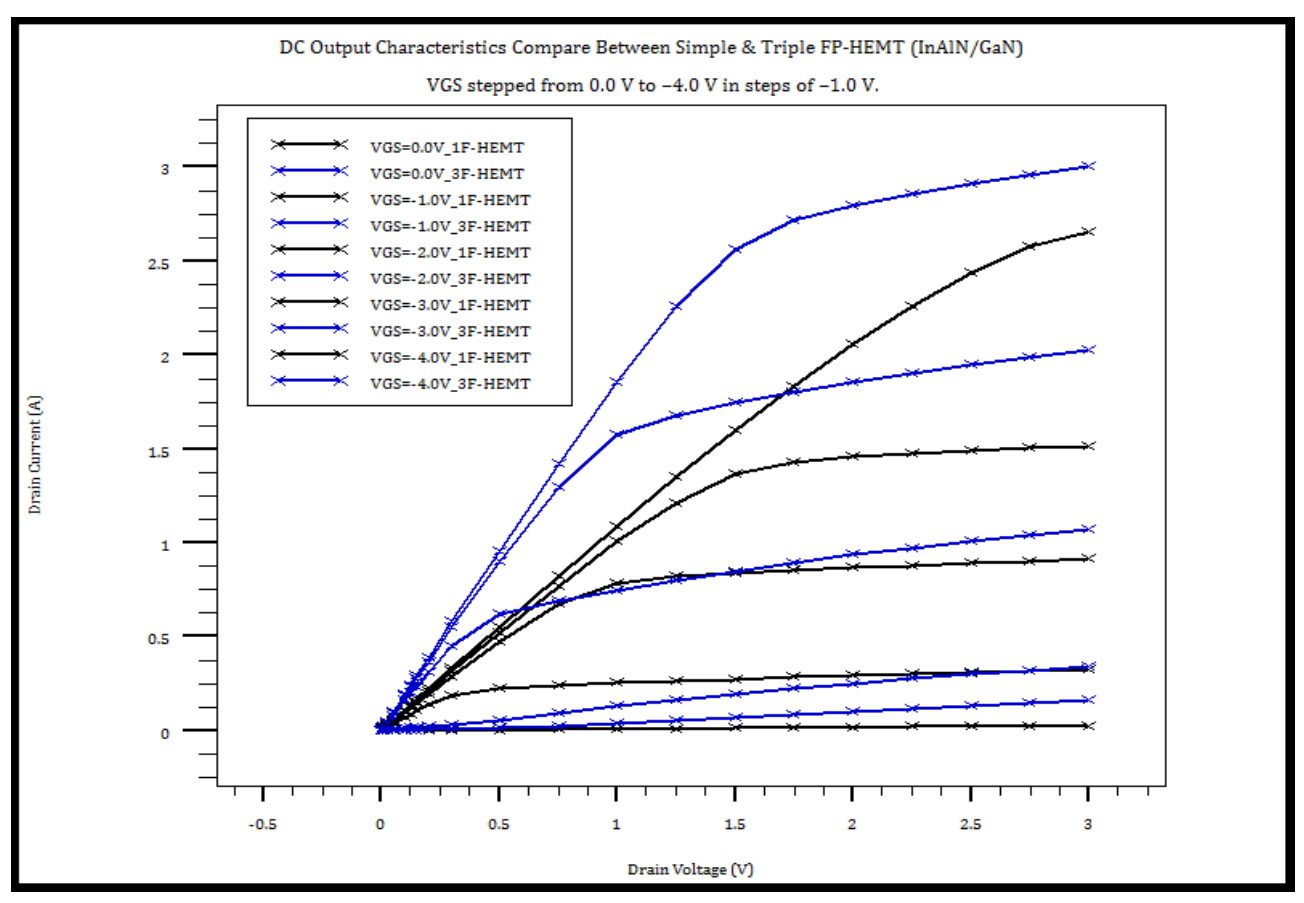

Figure 2. The transfer curve simulated with $\mathrm{V}_{\mathrm{DS}}$ fixed between $2 \mathrm{~V}$ to $\mathrm{V}_{\mathrm{DD}}$ and $\mathrm{V}_{\mathrm{GS}}$ sweeping from $1 \mathrm{~V}$ to $-10 \mathrm{~V}$, the DC Output characteristics of the InAlN/GaN HEMT with a gate length of $15 \mathrm{~nm}$ and $\mathrm{V}_{\mathrm{GS}}$ stepped from $1.0 \mathrm{~V}$ to $-4.0 \mathrm{~V}$ in steps of $-1.0 \mathrm{~V}$

The pinch-off voltage was found to be $-4.0 \mathrm{~V}$, as shown in Figure 2(b), which validates the suitability of Schottky contacts technology for InAlN/GaN heterostructure with improved device performance [27]. The extrinsic transconductance (gm) characteristics of the device, where the simulation is extracted displays a maximum peak gm as $840 \mathrm{mS} / \mathrm{mm}$ for $3 \mathrm{FP}$ and $560 \mathrm{mS} / \mathrm{mm}$ for SFP at $\mathrm{V}_{\mathrm{DS}}=5.0 \mathrm{~V}$. This peak appears in the curve of the transconductance as a dependence on the gate bias VGS. The simulated HEMT device noticeably reflects the DC behavior various operating conditions, which correspond to the 2DEG is affected by the presence of charged species from the channels modulated sheet density by different gate voltages. These performances are better quality of the values earlier reported for the device similar based on III-N material [28], [29].

The total parasitic resistance is generally dominated by a low Ohmic contact resistance [30] which is a large amount advantageous and could be attributed to the increased carrier concentration or/and an increased carrier mobility [31].

\subsection{Breakdown voltage}

The impact ionization-generation model applies in order to understand their impact on off-state breakdown voltages should be turned-on at techniques to simulate in the software process, this has used the impact Selb statement in which the severe impact ionization model is activated, here the beam statement is used to specify an optical source of carrier pair generation in addition to the thermal generation provided by recombination SRH (short read holes) GIGA and Blaze model.

In Thermal effect must be taken of the hot electrons, lattice variations of the exterior temperature and self heating of device into account [23]. It is based on this work on the model of Wachutka [32] and includes all thermal sources and sinks (Joule heat, Thomson term, etc.). Successful thermal modeling requires appropriate boundary conditions to be specified.

The off-state $\mathrm{I}_{\mathrm{DS}}-\mathrm{V}_{\mathrm{DS}}$ characteristics or breakdown voltage of conventional InAlN/GaN HEMTs with a wide drain bias region of the gate voltage between $-1 \mathrm{~V}$ and $-4 \mathrm{~V}$ are shown in Figure 3 . The conventional HEMTs demonstrate the off-state breakdown voltages of a $180 \mathrm{~V}$ and $875 \mathrm{~V}$. Growth of the AIN layer was particularly crucial indicated in that the breakdown characteristic of the HEMT device with this layer has been significantly improved in this device as well as their resistivity [33]. It is believed that enhancement of the off-state breakdown voltage of the HEMT device is attributed to a better carrier confinement and the increased back-barrier height of the AlN buffer layer used suppresses the spillover of the 
2DEG into the buffer layer and postpones the punch through of the buffer layer, thus can be particularly prominent as a function of the sub-threshold drain leakage current and increasing the breakdown voltage really very remarkable.

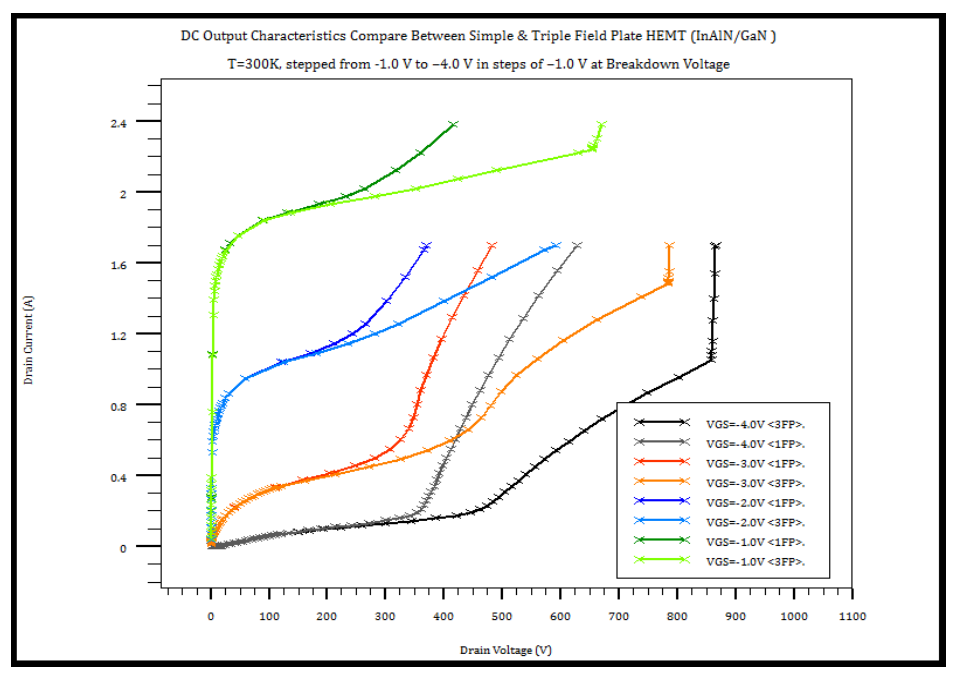

Figure 3. The DC Output characteristics of the InAlN/GaN HEMT with a gate length of $15 \mathrm{~nm}$ and $\mathrm{V}_{\mathrm{GS}}$ stepped from $-1.0 \mathrm{~V}$ to $-4.0 \mathrm{~V}$ in steps of $-0.5 \mathrm{~V}$ for breakdown voltage

At a certain drain current level value with increasing a channel pinch-off effect, and the punch through of the electrons into the buffer layer causes a rapid increase of the drain leakage current the punch through of the HEMT device breakdown be occurs [34], [35] and is known as the buffer-layer punch through effect [36], such as $200 \mathrm{~mA} / \mathrm{mm}$ in $\mathrm{V}_{\mathrm{GS}}=-4 \underline{\mathrm{V}}$ for example. As mentioned above, the electrons spilling over from the channel to the buffer layer at a higher drain supply voltage can from the buffer leakage current. The drain current change in $I_{D S}$ for the remarkable kink is maximum near the pinchoff of the device and reduces with the decline in the gate voltage.

\subsection{Kin effect}

Figure 4 shows the output characteristics of a 3FP-HEMT with Kink effect for temperature ambient. The device shows significant changes in the current density with a rise after stress with abrupt growth in current at different $V_{G S}$ voltage bias. In particular, it exhibits trapping/detrapping time constraints being researched vigorously indicating the existence of the traps in device [37].

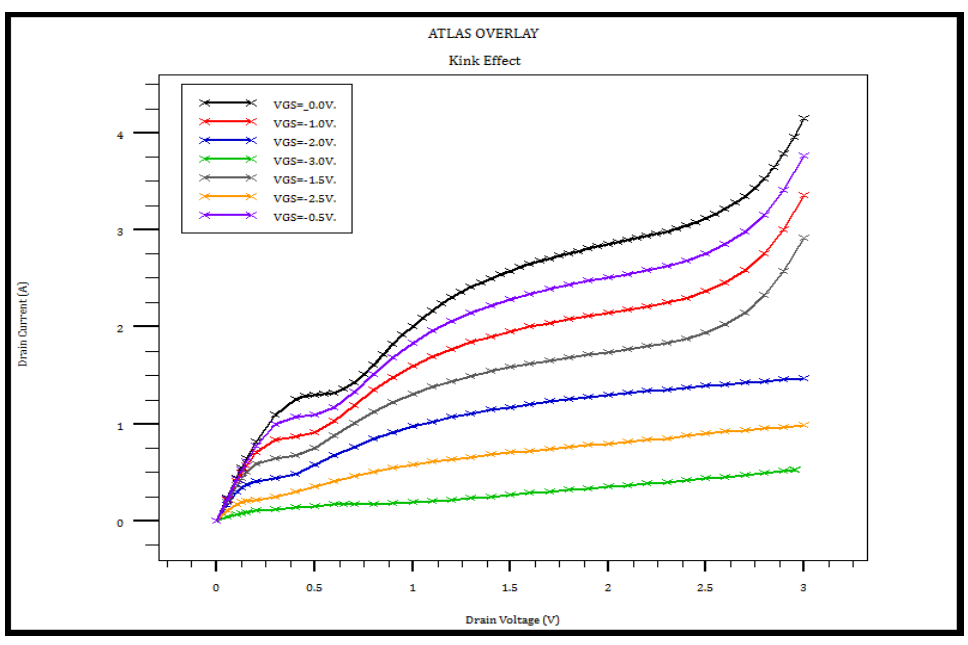

Figure 4. Output characteristics with kink effect 
Resulting in an accumulation of holes amending surface potentials or channel / substrate interface indicates the critical drain voltage $\left(\mathrm{V}_{\text {kink }}\right)$, this region of the Kink effect is highest in the output conductance. The study of the reliability of the Kink effect, especially with the gate bias Vkink increases regular in III- V materials, devices are field-assisted in nature and it is suggested that this kink could be induced by hot electron trapping and field-assisted de-trapping via donor-like traps in the buffer layer [38].

\subsection{AC results}

In this simulation, the maximum gain shown for the current $\mathrm{H} 21$ are between $67 \mathrm{~dB}$ for FP-HEMT and for others is $63 \mathrm{~dB}$ at $1 \mathrm{GHz}$, the maximum stable power gain is $38 \mathrm{~dB}$ for the first and for others is $37 \mathrm{~dB}$ at $1 \mathrm{GHz}$. Are shown in Figure 5.

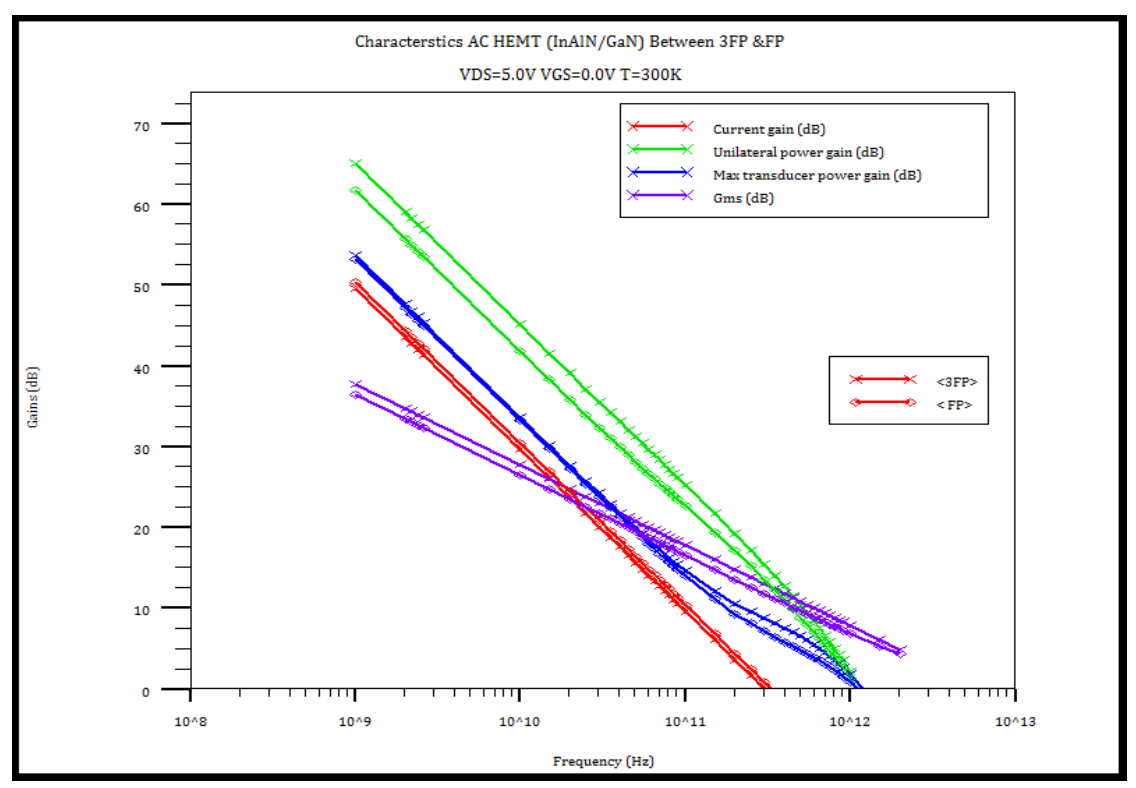

Figure 5. The simulated current gain $\left(\mathrm{H}_{21}\right)$ and maximum gain power stability (GMS) versus frequency for the $\mathrm{L}_{\mathrm{G}}=15 \mathrm{~nm}$ InAIN/GaN HEMTs. The bias conditions were $\mathrm{V}_{\mathrm{DS}}=2 \mathrm{~V}$ and $\mathrm{V}_{\mathrm{GS}}=0 \mathrm{~V}$

These result values were extracted from the extrinsic S-parameters and were then used to verify the intrinsic values of this device by simulation. An important advantage is optimized in the device; the electronic transfer in the channel due to the effect of the capacitances requires the high values of the gate to source capacitance $\left(\mathrm{C}_{\mathrm{GS}}\right)$, which result from the extended effective gate length [39]. We determined the cutoff frequency of the first device is $290 \mathrm{GHz}$ and $305 \mathrm{GHz}$ for another. The value of the maximum frequency is approximately $1.2 \mathrm{THz}$ with a slope $0 \mathrm{~dB} / \mathrm{Dec}$ [40].

The decline in the frequency value of the devices is fundamentally associated with the length of the gate, as well as the design of the device, and therefore the presence of the field plate has contributed to this reduction in value of the cutoff frequency. For comparison, the highest cutting frequency reported in nitride transistors to date was $670 \mathrm{GHz}[41]$ and $1 \mathrm{THz}[42]$.

\section{CONCLUSION}

We have presented the power performance of a $15 \mathrm{~nm}$ gate length InAlN/GaN HEMTs on $\mathrm{SiC}$ substrates using the periphery oxide of the gate for structures with and without a field plate. These devices exhibited current density as high as $2.05 \mathrm{~A} / \mathrm{mm}$, a peak extrinsic transconductance of $840 \mathrm{mS} / \mathrm{mm}$ for $3 \mathrm{FP}$ and $560 \mathrm{mS} / \mathrm{mm}$ for SFP at $\mathrm{V}_{\mathrm{DS}}=5.0 \mathrm{~V}$, and a cutoff in the first device is $290 \mathrm{GHz}$ and $305 \mathrm{GHz}$ for other. The maximum frequency was $1.2 \mathrm{THz}$, maximum breakdown voltage of a 180 and $875 \mathrm{~V}$. These results demonstrate the possibility of using this technology in different application with this optimized structure. 


\section{ACKNOWLEDGEMENTS}

The authors acknowledge the Agency space algerian are supported this work by through the Satellite development center

\begin{tabular}{|c|c|}
\hline \multicolumn{2}{|l|}{ Acronyms } \\
\hline ITER & Iteration steps \\
\hline $\mathrm{dt}$ & Denote differential interval of quantity \\
\hline dir & The direction primary injection direction for the contact \\
\hline BOUNDP & $\begin{array}{l}\text { Defines the length units for all positional bound } \\
\text { parameters }\end{array}$ \\
\hline TSTEP, XSTEP & $\begin{array}{l}\text { Denote integer multiples of the corresponding } \\
\text { differential intervals }\end{array}$ \\
\hline AGDEVICE & Algorithm genetic device \\
\hline QREGION & Quantum region definition \\
\hline SCHRREGION & Schrödinger region definition \\
\hline
\end{tabular}

\section{REFERENCES}

[1] W. W. J. X. D. C. Z. J. S., and S. R. L. Shunpu, "Electrical/optical dual-function redox potential transistor", Electronic Properties And Materials," Scientific Reports, vol. 3, n 3391, 2013.

[2] J. R. W. C., and C. P. S. Tucker, "Silicon field-effect transistor based on quantum tunnelling," Appl. Phys. Lett, vol. 65, pp. 618-620, 1994.

[3] W. Shockley, "A unipolar field-effect transistor," Proc. IRE 1952, IEEE, vol. 40, n 11, p. 1365, 2007.

[4] D. e. a. Nilsson, "Bi-stable and dynamic current modulation in electrochemical organic transistors," Adv. Mater, vol. $14, \mathrm{n}^{\circ} 51,2002$.

[5] S. B. D. W. K. S. B. H. D. Z. a. C. M. K. S. Boutros, "Normally-off 5A/1100V GaN-on-silicon device for high voltage applications," in IEDM Tech. Dig, no 751-753, 2009.

[6] e. a. K. Kobayashi, "New slant on field plates for gallium nitride HEMTs," Appl. Phys. Express, vol. 7, 2014.

[7] T. N. Y. K. Y. S. K. T. I. O. a. M. Y. W. Saito, "On-resistance modulation of high voltage GaN HEMT on sapphire substrate under high applied voltage,” IEEE Electron Device Lett, vol. 28, nº 8, p. 676-678, 2007.

[8] M. J.-M. M. L. M. a. S. H. Y. Wu, "A 97.8\% efficient GaN HEMT boost converter with 300-W output power at 1 MHz,”" IEEE Electron Device Lett., vol. 29, nº 8, p. 824-826, 2008.

[9] A. S. M. M. R. P. S. S. S. P. M. C. T. W. U. K. M. a. P. P. Y. Wu, " $30 \mathrm{~W} / \mathrm{mm}$ GaN HEMTs by field plate optimization,", IEEE Electron Device Lett, vol. 25, n 3, p. 117-119, 2004.

[10] T. D. T. P. H. Phuong, "Genetic Algorithm for Optimization of HEMT Model Parasitic Parameters," International Conference on Electronic Computer Technology, p. 600 - 603, 2009.

[11] D. R. S. S. S. K. R. L. R. K. C. S. V. a. R. K. S. Amit, "Design and Fabrication of Multi-finger Field Plate for Enhancement of AlGaN/GaN HEMT Breakdown Voltage," Defence Science Journal, vol. 68, $\mathrm{n}^{\circ} 3$, pp. 290-294, 2018.

[12] K. V. a. R. M. M. Arundevi, "Real Coded Genetic Algorithm Based Improvement of Efficiency in Interleaved Boost Converter," International Journal of Power Electronics and Drive Systems (IJPEDS), vol. 5, $\mathrm{n}^{\circ} 4$, p. 529, 2018.

[13] R. E. T. N. A. E.-B. S. K. E.-L. \&. W. M. M. M. E. Yahia, "Rogue waves lead to the instability in GaN semiconductors," Scientific Reports, vol. 3, n 12245, 2015.

[14] G. B. S. K. S. S. Shee, "Quantum Analytical Modeling for Device Parameters and I - V Characteristics of Nanoscale Dual-Material Double-Gate Silicon-on-Nothing MOSFET," IEEE Transactions on Electron Devices, vol. 61, no 8, p. $2697-2704,2014$.

[15] K. T. S. K. K.F. Man, “Genetic algorithms: concepts and applications [in engineering design,” Trans. On Industrial Electronics, vol. 43, pp. 519-534, 1996.

[16] C. F. G. E. Programming, "Mathematical Modeling by an Artificial Intelligent," ISBN 972-95890-5-4, 2002.

[17] T. A. D. L. I. P. R. F. C. P. G. a. G. M. G. V. Resta, "Scaling trends and performance evaluation of 2-dimensional polarity-controllable FETs," Scientific Reports, p. PMC5372079, 2017.

[18] S. M. E.-G. Y. A. Hussein, "Modelling and optimization of microwave devices and circuit using genetic algorithm," IEEE Transaction on Microwave Theory and Techniques, vol. 52, pp. 329-336, 2004.

[19] M. I. o. T. (. a. IQE, "RF LLC have used an aluminum gallium nitride (AlGaN) back-barrier to improve the frequency performance of indium aluminum nitride (InAlN) high-electron-mobility transistors (HEMTs)," [Dong Setup Lee et al, IEEE Electron Device Letters, 2011.

[20] F. Laboratories, Fujitsu Develops World's First Gallium-Nitride HEMT for Power Supply, Japan: Kawasaki, 2009.

[21] P. P. R. M. M. R. K. Yadav, "TCAD Simulations and Small Signal Modeling of DMG AlGaN/GaN HFET," International Journal of Electrical and Computer Engineering (IJECE), vol. 7, no 4, pp. 1839-1849, 2017.

[22] J. X. ,. N. U. G. Krokidis, "A fully 2-dimensional, quantum mechanical calculation of short-channel and drain induced barrier lowering effects in HEMTs," Solid-State Electronics, vol. 52, p. 625-631, 2008.

Int J Pow Elec \& Dri Syst, Vol. 10, No. 1, March 2019 : 398 - 405 
[23] S. v. corporation, "GaN HEMT Breakdown," Tcad-Silavaco, 2014. [Online]. Available: https://www.silvaco.com/examples/tcad/section20/example2/index.html.

[24] J. X. N. U. Krokidis, "A fully 2-dimensional, quantum me chanical calculation of short-channel and drain induced barrier lowering effects in HEMTs," Solid-State Electronics, vol. 52, p. 625-631, 2008.

[25] P. Papadopoulouy, 2013. [Online]. Available: http://hephaestus.teikav.edu.gr/index.php/ct-menu-item-7/electricalengineering/226-device-simulations.

[26] S. H. Q. J. S. Z. Tang e C. L. a. K. J. C. Liu, "600 V High-Performance AlGaN/GaN HEMTs with AlN/SiNx Passivation," New Orleans, Louisiana, USA.: CS MANTECH Conference, May 13th - 16th, (2013).

[27] S. Y. W. C. B. Z. Z. F. S. C. K. J. C. Q. Zhou, "High voltage InAlN/GaN HEMTs with nonalloyed Source/Drain for RF power applications," Solid-State Electronics, vol. 91, pp. 19-23, 2014.

[28] J. C. Z. Y. W. H. H. Z. J. F. Z. a. Y. H. J. S. Xue, "Fabrication and characterization of InAlN/GaN-based doublechannel high electron mobility transistors for electronic applications," J. Appl. Phys, vol. 111, $\mathrm{n}^{\circ}$ 114513, 2012.

[29] C. C. J. Z. e. a. B. Tian, "Structure and electrical characteristics of AlGaN/GaN MISHFET with Al2O3 thin film as both surface passivation and gate dielectric," Semicond Sci Technol, no 085023, p. 26, 2011.

[30] K. K. K. \&. M. A. Y. Awano, "Short-channel effects in sub quarter-micrometer-gate HEMT's: Simulation and experiment," IEEE Transactions on Electron Devices , vol. 36, n 10, pp. 2260 - 2266, 1989.

[31] N. Dubuc, "Electro thermal modeling SiC MESFET transistors and optimization of an architecture for the Doherty power amplifier with high efficienc," France: Ph.D. Thesis University of Limoges, 2003.

[32] D. V. M. C. V. M. R. G. B. F. I. A. S. L. Z. M. Valdinoci, Impact-ionization in silicon at large operating temperature, Kyoto, Japan: SISPAD '99, 1999.

[33] M. W. a. K. J. Chen, "Kink Effect in AlGaN/GaN HEMTs Induced by Drain and Gate Pumping," IEEE Electron Device Letters, vol. 32, no 4, p. 483, 2011.

[34] Y. C. H. J. K. M. L. C. Liu, "Optimization of a Common Buffer Platform for Monolithic Integration of InGaN/GaN Light-Emitting Diodes and AlGaN/GaN High-Electron-Mobility Transistors," Journal of Electronic Materials, vol. $45, \mathrm{n}^{\circ}$ 5, p. 2092-2101, 2016.

[35] H. O. B. F. e. a. Bahat-Treidel E, "Punch through-voltage enhancement of AlGaN/GaN HEMTs using AlGaN double heterojunction confinement," IEEE Trans Electron Devices, vol. 12, p. 55, 2008.

[36] B. B. A. G. M. K. Z. Kourdi, "Side Effects in InAlN/GaN High Electron Mobility Transistors," Microelectronic Engineering, vol. 142, pp. 52-57, 2015.

[37] E. G. J. L. a. M. H. S. Nuttinck, "Floating-body effect in Al-GaN / GaN HEMT power," Proceeding of GaAs Symposium, pp. 33-36, 2002.

[38] S. C. T. P. M. J. Yang, Determination of trap energy levels in AlGaN/GaN HEMT, Notre Dame USA: 71st Annual 1548-3770, 2013.

[39] E. H. M. C. a. T. P. W. Chung, "AlGaN/GaN HEMT With 300-GHz fmax," IEEE Electron Device Letters, vol. 31, p. 197, 2010.

[40] I. I. Z. Y. J. S. S. Ghandi, "0.5 GHz-1.5 GHz Bandwidth 10W GaN HEMT RF Power Amplifier Design," International Journal of Electrical and Computer Engineering (IJECE), vol. 8, nº 3, 2018.

[41] M. X. W. Y. L. P.-H. L. L.-S. Z. J. L. K. R. V. D. M. X. W. Y. L. P.-H. L. L.-S. J. Z. K. L. V. R. W. D. R. L. S. Sarkozy, "Sub-50nm indium phosphide high electron mobility transistor technology for terahertz monolithic microwave integrated circuits and systems," International Conference Indium Phosphide and Related Materials (IPRM), pp. 1-2, 2013.

[42] T. lee, "Terahertz electronics :Opportunities, challenges and technologies," International Symposium VLSI Design, Automation, and Test (VLSI-DAT), vol. 1, 2013. 